\title{
Remote ischemic postconditioning confers neuroprotective effects via inhibition of the BID-mediated mitochondrial apoptotic pathway
}

\author{
XIAOYING GAO, YUN LIU, YUYING XIE, YING WANG and SIHUA QI \\ Department of Anesthesiology, The Fourth Affiliated Hospital of Harbin Medical \\ University, Harbin, Heilongjiang 150001, P.R. China
}

Received February 4, 2016; Accepted February 15, 2017

DOI: $10.3892 / \mathrm{mmr} .2017 .6652$

\begin{abstract}
Ischemic postconditioning has been demonstrated to alleviate brain ischemia/reperfusion-induced neuronal apoptosis; however, the protective mechanisms underlying the improved and more convenient method of remote ischemic postconditioning (RIPostC) are only recently beginning to be elucidated. Mitochondria are important in the regulation of cell apoptosis, and the B-cell lymphoma 2 (Bcl-2) homology 3 interacting-domain death agonist (BID) promotes the insertion/oligomerization of $\mathrm{Bcl}-2$-associated $\mathrm{X}$ protein into the mitochondrial outer membrane, leading to the release of proapoptotic proteins from the mitochondria. The present study hypothesized that RIPostC targets the BID-mediated mitochondrial apoptotic pathway to exert neuroprotective effects, and the optimal time window for RIPostC application was investigated. RIPostC was conducted as follows: Three 10-min cycles of bilateral femoral artery occlusion with intervals of 10 min reperfusion after 0,10 or $30 \mathrm{~min}$ of brain reperfusion. The results revealed that reperfusion induced significant activation of BID, via proteolytic cleavage and translocation to the mitochondria, as determined using western blot analysis and immunofluorescence staining. Mitochondrial release of cytochrome $c$ was additionally detected during BID activation, all of which were inhibited by the application of RIPostC. When RIPostC was applied during reperfusion, it demonstrated a significant protective effect. Furthermore, the infarct volume, neurological function and the degree of neuronal apoptosis were improved with application of RIPostC. These results suggested that the protective mechanisms of RIPostC may be associated with inhibition of the BID-mediated mitochondrial
\end{abstract}

Correspondence to: Dr Sihua Qi, Department of Anesthesiology, The Fourth Affiliated Hospital of Harbin Medical University, 37 Yiyuan Street, Harbin, Heilongjiang 150001, P.R. China

E-mail: qisihua2007@sina.com

Key words: remote ischemic postconditioning, neuroprotection, $\mathrm{BH} 3$ interacting-domain death agonist, apoptotic apoptotic pathway, which may act as a potential molecular target for therapeutic intervention in the future.

\section{Introduction}

Thrombolytic therapy following the onset of stroke has been demonstrated to prevent apoptosis of neural cells at risk of injury; however, the re-establishment of blood circulation may contribute to delayed secondary brain damage (1). Our previous study demonstrated that reperfusion induced mitochondrial ultrastructural alterations and dysfunction (2); mitochondria are important in the regulation of intrinsic apoptosis (3). Previous in vivo and in vitro models have demonstrated that postconditioning significantly inhibits neuronal apoptosis and protects the brain from reperfusion injury $(4,5)$. Therefore, it appears that inhibition of neuronal apoptosis is important in postconditioning-initiated neuroprotection. However, the mechanisms by which remote ischemic postconditioning (RIPostC) protects neurons from reperfusion injury and the optimal application time of RIPostC remain to be elucidated, in order for this method to be translated into clinical practice.

The B-cell lymphoma 2 ( $\mathrm{Bcl}-2)$ family of genes regulates apoptosis and consists of two groups: Proapoptotic and anti-apoptotic proteins. The ratio between proapoptotic and anti-apoptotic members of the Bcl-2 family determines the fate of the cell (6). Bcl-2 homology 3 (BH3) interacting-domain death agonist (BID) is a member of the BH3-only subfamily, which is associated with proapoptotic proteins. It has previously been demonstrated that BID may bridge the crosstalk between extrinsic and intrinsic apoptotic pathways via its cleavage by caspase-8 (7). When cleaved, truncated BID (tBID) promotes apoptosis by activating Bcl-2-associated $\mathrm{X}$ protein (Bax) and eliciting mitochondrial outer membrane permeabilization (8). The infarct sizes and apoptotic indexes in $\mathrm{bid}^{-/-}$brains are greatly reduced under reperfusion conditions (9). BID is important in reperfusion-induced neuronal apoptosis, and postconditioning may significantly inhibit the apoptosis induced by ischemia/reperfusion injury. Furthermore, RIPostC may share common mechanistic signaling pathways with conventional postconditioning methods, although these are performed in ischemic organs and not in distal limbs (10). Therefore, the present study hypothesized that the BID-mediated 
mitochondrial apoptotic pathway is an important target for $\mathrm{RIPostC}$ to prevent reperfusion-induced neuronal apoptosis.

\section{Materials and methods}

Animal and experimental groups. All animal protocols used in the present study were approved by the Harbin Medical University Committee on the Guidelines for Animal Experiments (Harbin, China). All rats were handled according to the Guidelines for the Care and Use of Laboratory Animals of the National Institutes of Health. Adult male Sprague-Dawley rats (129 total; weight, 260-280 g; age, 6-8 weeks) were provided by the laboratories of The Second Affiliated Hospital of Harbin Medical University (Harbin, China), and housed under controlled conditions (temperature, $27 \pm 2^{\circ} \mathrm{C}$; humidity, 60-70\%) under a 12 -h light/dark cycle. The rats were allowed ad libitum access to food and water. Animals were randomly divided into five groups (sham, 5 rats; control, 49 rats; R-0, 49 rats; R-10, 13 rats; R-30, 13 rats), according to the RIpostC starting times: The sham group, the control group (ischemia, $120 \mathrm{~min}$ ), and three RIPostC groups. In the three RIPostC groups, RIPostC was conducted as follows: Three 10-min cycles of bilateral femoral artery occlusion with an interval of $10 \mathrm{~min}$ reperfusion after 0,10 or $30 \mathrm{~min}$ of brain reperfusion (R-0, R-10 and R-30 groups, respectively), following administration of local anesthesia. The protocol for RIPostC in each group is schematically presented in Fig. 1A. In order to exclude the effect of RIPostC on the expression of apoptosis-related proteins in non-ischemic reperfusion period, a RIPostC + sham group was added as an additional control in the western blot analysis.

Transient middle cerebral artery occlusion (MCAO) model. Focal cerebral ischemia was induced by MCAO as previously described, with minor modifications (11). Briefly, 260-280 g male Sprague-Dawley rats were anesthetized by intraperitoneal injection of pentobarbital sodium $(50 \mathrm{mg} / \mathrm{kg}$ in normal saline), and a caudal ventral artery catheter was inserted for continuous arterial blood pressure monitoring. Subsequently, the left external carotid artery (ECA), internal carotid artery (ICA) and common carotid artery were exposed. A 4-0 monofilament nylon thread (40-3734 PK 10; Doccol Corporation, Sharon, MA, USA) with a silicon-rubber-coated tip was then inserted into the ICA from a hole in the ECA; the suture was advanced $16-18 \mathrm{~mm}$ through the ICA, until a mild resistance was felt for $120 \mathrm{~min}$. Blood flow was restored by gently withdrawing the suture. During these procedures, the rectal temperature was monitored continuously with a rectal probe; temperature was maintained at $37 \pm 0.5^{\circ} \mathrm{C}$ by the use of a lamp and pad. The mean arterial pressure and arterial blood gas values were recorded at 3 min prior to insertion of the suture (baseline), $60 \mathrm{~min}$ following MCAO (ischemia), and 10 min following reperfusion in each group. Rats in the sham group underwent the same procedure, with the exception of occlusion. Rats in the control group underwent ischemia/reperfusion without RIPostC. Prior to the induction of RIPostC, rats were allowed to regain consciousness, and the neurological deficit score was determined by a blinded observer based on Longa grade point standards (12). These standards were as follows: 0 , no neurological deficits; 1 , failure to fully extend the right forepaw; 2 , circling to the right; 3 , falling to the right; and 4, unable to walk spontaneously and exhibiting depressed levels of consciousness. In this experiment, the rats that scored 2 or 3 were adopted for further study.

Measurement of neurobehavioral scores and infarct volume. At $24 \mathrm{~h}$ after reperfusion, a revision of the 18-point scoring table proposed by Garcia et al (13) was used for neurobehavioral evaluation by a blinded observer. The 18-point scoring table was based on the following six tests: i) Spontaneous activity; ii) symmetry in the movement of four limbs; iii) forepaw outstretching; iv) climbing; v) body proprioception; and vi) response to vibrissal stimulation. The scores were summed up at the end of the evaluation (minimum score, 3; maximum score, 18). Following anesthesia with pentobarbital sodium $(30 \mathrm{mg} / \mathrm{kg})$, the mice were rapidly decapitated. The brains were frozen at $-20^{\circ} \mathrm{C}$ for $15 \mathrm{~min}$ and were then cut into five $2 \mathrm{~mm}$-thick coronal sections, which were stained with $2 \%$ 5-triphenyl-2H tetrazolium chloride (Sigma-Aldrich; Merck KGaA Millipore, Darmstadt Germany) at $37^{\circ} \mathrm{C}$ for $15 \mathrm{~min}$ (14). The infarct volume was analyzed using Image-Pro Plus software version 5.1 (Media Cybernetics Inc., Rockville, MD, USA). The volume of the infarct area was calculated as the percentage of the infarct volume relative to whole brain volume (15).

Terminal deoxynucleotidyl transferase dUTP nick end labeling (TUNEL) staining. Following $24 \mathrm{~h}$ of reperfusion, brain cell apoptosis in the peri-infarct cortex ( $n=5 /$ group) was assessed in situ via TUNEL staining, as previously described (16). The infarct core and peri-infarct zone were defined according to well-established protocols in rodent models of unilateral proximal MCAO (17) (Fig 1B). TUNEL staining was quantitatively evaluated using the method described by Wang et al (18). Positively stained cells were counted in 10 random peri-infarct cortexes per rat, and the total number of positively stained cells in these pixels was then counted and expressed as cells $/ \mathrm{mm}^{2}$.

Western blot analysis. At 3, 6, 12 and 24 h following reperfusion, the brain samples were collected, and protein isolation from cortical tissues, based on total cell extracts or subcellular fractionation (cytosolic), was performed as previously described (19). A total of $20 \mathrm{mg}$ tissue was homogenized and lyzed in radioimmunoprecipitation assay buffer on ice and the protein concentration of samples was determined using the Bradford protein assay kit (both from Beyotime Institute of Biotechnology, Shanghai, China). Western blotting was performed as previously described (20), and the following primary antibodies were incubated at $4^{\circ} \mathrm{C}$ overnight: anti-BID (1:200; cat. no. sc-6358; Santa Cruz Biotechnology, Inc., Dallas, TX, USA), anti-cytochrome $c$ (1:300; cat. no. ab13575) and anti- $\beta$-actin (1:2,000; cat. no. ab8226) (both from Abcam, Cambridge, UK). The polyvinylidene fluoride membranes were incubated with horseradish peroxidase-conjugated rabbit anti-goat immunoglobulin (Ig) G (1:1,000; cat. no. ZB-2306) and goat anti-mouse IgG secondary antibodies (1:1,000; cat. no. ZB-2305) (both from ZSGB-BIO, Beijing, China) for $1 \mathrm{~h}$ at room temperature. The expression of $\beta$-actin in the same membrane served as an internal reference. Blots were detected 

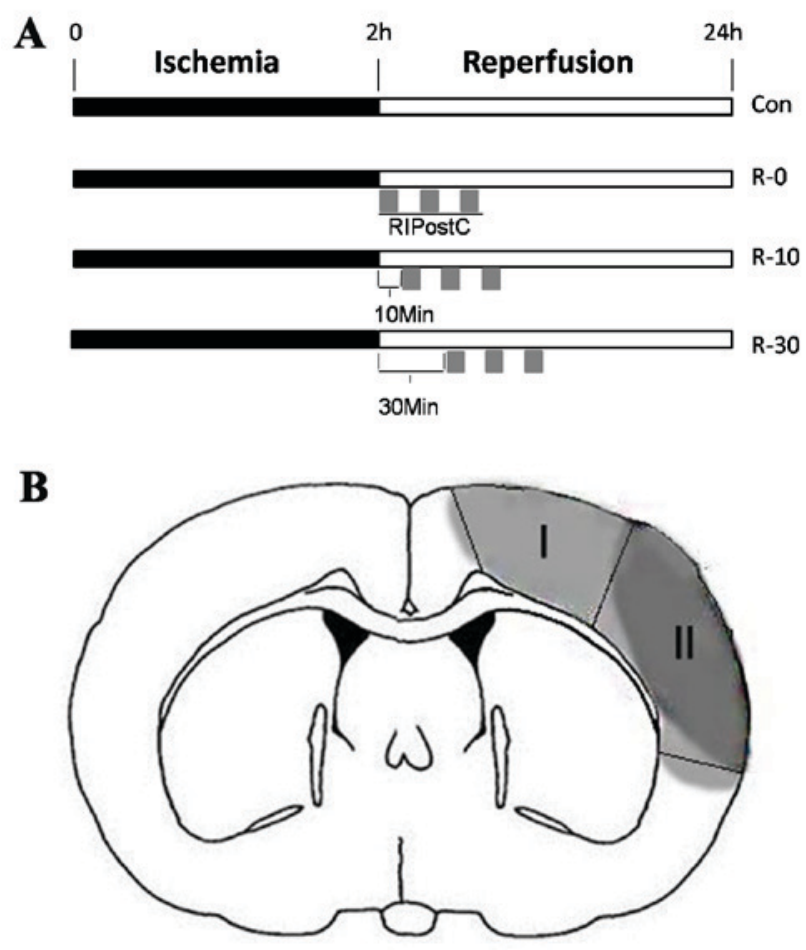

Figure 1. (A) Schematic protocol for RIPostC. Control group rats underwent ischemia for $2 \mathrm{~h}$ and reperfusion for $24 \mathrm{~h}$. Rats in the RIPostC groups underwent three cycles of 10-min occlusion/10-min release of the bilatera femoral artery (gray rectangles) during reperfusion. Rats were divided into three RIPostC groups: R-0, R-10 and R-30, according to the starting time of RIpostC (0, 10 and 30 min post-reperfusion). (B) Representative coronal section through anterior mid-caudate and anterior hippocampus level (top to bottom panel). The light gray area (region I) is defined as the ischemic peri-infarct zone, and the dark gray area (region II) is defined as the ischemic core. RIPostC, remote ischemic postconditioning.

using a luminescent image analyzer and semi-quantified using Image Quant TL version 7.0 (both from GE Healthcare Biosciences, Pittsburgh, PA, USA).

Immunofluorescence analysis. Coronal sections were used for immunofluorescence staining. The procedures for immunofluorescent staining were conducted as previously described (11). Briefly, the sections were first blocked with 5\% bovine serum albumin (BSA; Beyotime Institute of Biotechnology) for $30 \mathrm{~min}$ followed by overnight incubation at $4^{\circ} \mathrm{C}$ with the primary antibody, anti-BID (1:100; cat. no. sc-6358; Santa Cruz Biotechnology, Inc.), diluted in BSA. After washing three times with PBS, sections were incubated with a secondary antibody (1:300; cat. no. bs-0294M-RBITC; BIOSS, Beijing, China) conjugated to a fluorochrome at room temperature for $1 \mathrm{~h}$. Following the final wash, the sections were placed on coverslips using a fluorescent mounting medium (Beyotime Institute of Biotechnology). Double immunofluorescent staining was conducted in a linear fashion as previously described (21). Following staining with the first set of primary and secondary antibodies as aforementioned, the second set of primary antibodies used were as follows: anti-cytochrome $c$ oxidase (COX)IV (1:200; cat. no. ab153709) and anti-neuronal nuclear antigen (NeuN; 1:500; cat. no. ab177487) (both from Abcam) and corresponding secondary antibodies (1:400; cat. no. bs-0295d-FITC; BIOSS) conjugated to a different fluorochrome, were applied in the same manner as previously described. This procedure was conducted with caution, to prevent cross-reaction of the secondary antibodies with the two sets of primary antibodies, which were prepared in various species. DAPI was used as a nuclear counterstain. Images were obtained by fluorescence microscopy (Nikon Corporation, Tokyo, Japan) and microscopic examination was conducted at excitation wavelengths of $540 \mathrm{~nm}$, and emission wavelengths of $605 \mathrm{~nm}$ (for red fluorochrome) and 465/515 nm (for green fluorochrome).

Statistical analysis. Statistical analyses were conducted using SPSS software, version 21.0 for Windows (IBM SPSS, Armonk, NY, USA). Data are presented as the mean \pm standard deviation of 3 independent experiments. One-way analysis of variance was conducted to determine significance followed by Bonferroni's post hoc correction for multiple comparisons. $\mathrm{P}<0.05$ was considered to indicate a statistically significant difference.

\section{Results}

\section{RIPostC mediates neuroprotective effects}

Infarct volume and neuroethology score. The present study did not detect any significant differences among the various time points for any of the physiological parameters, including rectal temperature, mean arterial pressure and arterial blood gas tension (Table I). It was demonstrated that RIPostC applied at the moment of reperfusion significantly reduced infarct volume in MCAO rats. All RIPostC groups, with the exception of the R-30 group (33.80 4 4.22\%), exhibited significantly reduced infarct volumes at $24 \mathrm{~h}$ following reperfusion

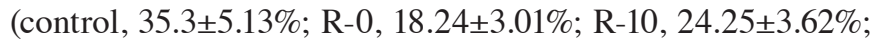
$\mathrm{P}<0.05$; Fig. $2 \mathrm{~A}$ and $\mathrm{B}$ ), this effect was particularly evident in the R-0 group. In addition, a similar result was observed with regards to neuroethology score; the 18-point scores of rats in the R-0 and R-10 groups were significantly higher compared with in the control group. Furthermore, the R-0 group exhibited significantly higher neurological scores compared with the R-10 group (control, 9.67 $\pm 0.81 ; \mathrm{R}-0,14.66 \pm 0.62 ; \mathrm{R}-10$, $12.83 \pm 0.68$; R-30, 9.83 \pm 0.72 ; $\mathrm{P}<0.05$; Fig. 2 C).

TUNEL-positive cells. The present study aimed to determine whether RIPostC is associated with a decrease in neuronal apoptosis in the peri-infarct cortex. TUNEL staining was nearly negative in the sham group; however, a large number of TUNEL-positive cells was observed in the control and R-30 groups. Only a few TUNEL-positive cells were observed in other RIPostC groups, in particular, very few TUNEL-positive cells were detected in the R-0 group (Fig. 3).

These results indicated that following application of RIPostC at the early stage of reperfusion infarct volume decreased, neuroethology score improved and the rate of apoptosis was reduced; these effects were particularly evident in the R-0 group.

\section{RIPostC-mediated effects on tBID and cytochrome $c$ levels} Expression of $t B I D$ and cytochrome $c$. The R-0 group was selected to explore the potential mechanism underlying the effects of RIPostC. Western blot analysis revealed that tBID 
Table I. Physiological parameters.

\begin{tabular}{|c|c|c|c|c|c|}
\hline \multirow[b]{2}{*}{ Parameter } & \multicolumn{5}{|c|}{ Group } \\
\hline & Sham & Con & $\mathrm{R}-0$ & $\mathrm{R}-10$ & $\mathrm{R}-30$ \\
\hline \multicolumn{6}{|c|}{ Temperature $\left({ }^{\circ} \mathrm{C}\right)$} \\
\hline Baseline & $37.00 \pm 0.10$ & $36.90 \pm 0.30$ & $36.80 \pm 0.20$ & $37.00 \pm 0.10$ & $36.90 \pm 0.10$ \\
\hline Ischemia & $36.90 \pm 0.20$ & $36.80 \pm 0.20$ & $36.80 \pm 0.10$ & $36.90 \pm 0.10$ & $36.80 \pm 0.20$ \\
\hline Reperfusion & $37.00 \pm 0.10$ & $36.80 \pm 0.10$ & $37.00 \pm 0.10$ & $36.80 \pm 0.20$ & $36.80 \pm 0.30$ \\
\hline \multicolumn{6}{|l|}{ MAP (mmg) } \\
\hline Baseline & $96.00 \pm 5.00$ & $96.00 \pm 4.00$ & $99.00 \pm 1.00$ & $98.00 \pm 3.00$ & $96.00 \pm 4.00$ \\
\hline Ischemia & $97.00 \pm 3.00$ & $98.00 \pm 2.00$ & $97.00 \pm 3.00$ & $97.00 \pm 5.00$ & $95.00 \pm 5.00$ \\
\hline Reperfusion & $98.00 \pm 2.00$ & $99.00 \pm 2.00$ & $96.00 \pm 3.00$ & $95.00 \pm 5.00$ & $98.00 \pm 2.00$ \\
\hline \multicolumn{6}{|l|}{$\mathrm{PaO}_{2}(\mathrm{mmH})$} \\
\hline Baseline & $165.00 \pm 3.00$ & $161.00 \pm 5.00$ & $163.00 \pm 3.00$ & $159.00 \pm 8.00$ & $162.00 \pm 3.00$ \\
\hline Ischemia & $159.00 \pm 7.00$ & $163.00 \pm 4.00$ & $162.00 \pm 5.00$ & $161.00 \pm 6.00$ & $165.00 \pm 2.00$ \\
\hline Reperfusion & $160.00 \pm 4.00$ & $162.00 \pm 7.00$ & $164.00 \pm 3.00$ & $167.00 \pm 1.00$ & $159.00 \pm 9.00$ \\
\hline \multicolumn{6}{|c|}{$\mathrm{PaCO}_{2}(\mathrm{mmHg})$} \\
\hline Baseline & $37.00 \pm 4.00$ & $38.00 \pm 2.00$ & $37.00 \pm 4.00$ & $38.00 \pm 2.00$ & $37.00 \pm 2.00$ \\
\hline Ischemia & $36.00 \pm 3.00$ & $36.00 \pm 4.00$ & $36.00 \pm 6.00$ & $36.00 \pm 5.00$ & $37.00 \pm 3.00$ \\
\hline Reperfusion & $37.00 \pm 3.00$ & $37.00 \pm 2.00$ & $36.00 \pm 5.00$ & $37.00 \pm 3.00$ & $38.00 \pm 1.00$ \\
\hline \multicolumn{6}{|l|}{$\mathrm{pH}$} \\
\hline Baseline & $7.40 \pm 0.02$ & $7.38 \pm 0.01$ & $7.39 \pm 0.01$ & $7.38 \pm 0.02$ & $7.36 \pm 0.03$ \\
\hline Ischemia & $7.39 \pm 0.01$ & $7.37 \pm 0.02$ & $7.39 \pm 0.01$ & $7.37 \pm 0.02$ & $7.38 \pm 0.01$ \\
\hline Reperfusion & $7.38 \pm 0.02$ & $7.39 \pm 0.01$ & $7.38 \pm 0.02$ & $7.38 \pm 0.01$ & $7.36 \pm 0.02$ \\
\hline
\end{tabular}

A

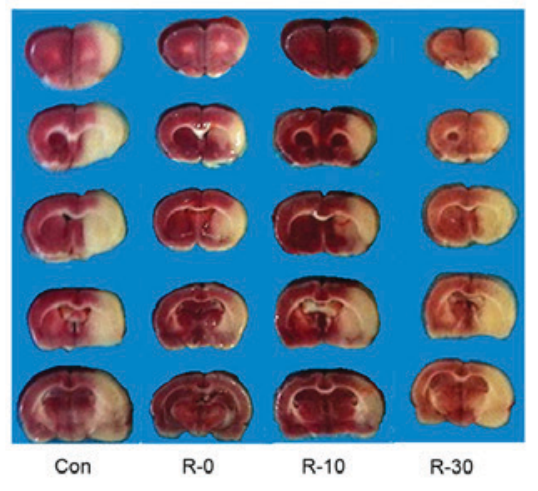

B

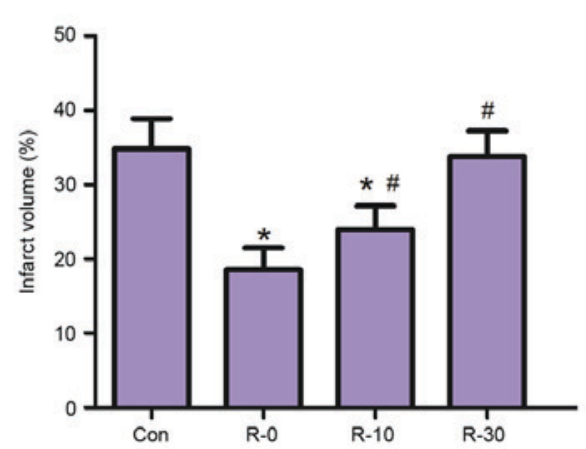

C

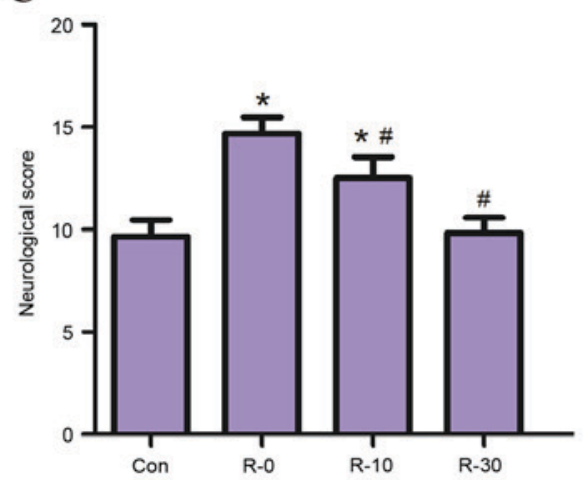

Figure 2. (A) Representative brain slices stained by tetrazolium chloride in the control and RIpostC groups. (B) Quantitative evaluation of infarct volumes in the various groups. (C) Neurological score of transient middle cerebral artery occlusion in the control and RIPostC groups. Neurological scores were evaluated at $24 \mathrm{~h}$ following reperfusion using the Garcia scoring system. Data are presented as the mean \pm standard deviation ( $\mathrm{n}=8 /$ group). ${ }^{\text {"P }}<0.05$ vs. the control group; ${ }^{\text {"P}} \mathrm{P}<0.05$ vs. R-0 group. Con, control; RIPostC, remote ischemic postconditioning; R-0, -10, -30, RIPostC 0, 10 and 30 min groups, respectively.

was readily detected in the control group brains $3 \mathrm{~h}$ following reperfusion, peaked at $6 \mathrm{~h}$ and was continuously detectable through the first $12 \mathrm{~h}$. The R-0 group exhibited a similar trend, however tBID expression was significantly reduced at each time point compared with in the control group (Fig. 4A and B). Cytochrome $c$ release into the cytosolic fraction was detected in the R-0 and control groups $3 \mathrm{~h}$ following reperfusion and at various time points throughout the first $24 \mathrm{~h}$. However, its expression was markedly reduced in the R-0 group compared with in the control group (Fig. 4C and D). In the RIPostC + sham group, the expression levels of tBID and cytochrome $c$ were similar to those in the sham group. The peak expression of tBID and cytochrome $c$ was significantly decreased in the R-0 group compared with control group (Fig. 5). 
A

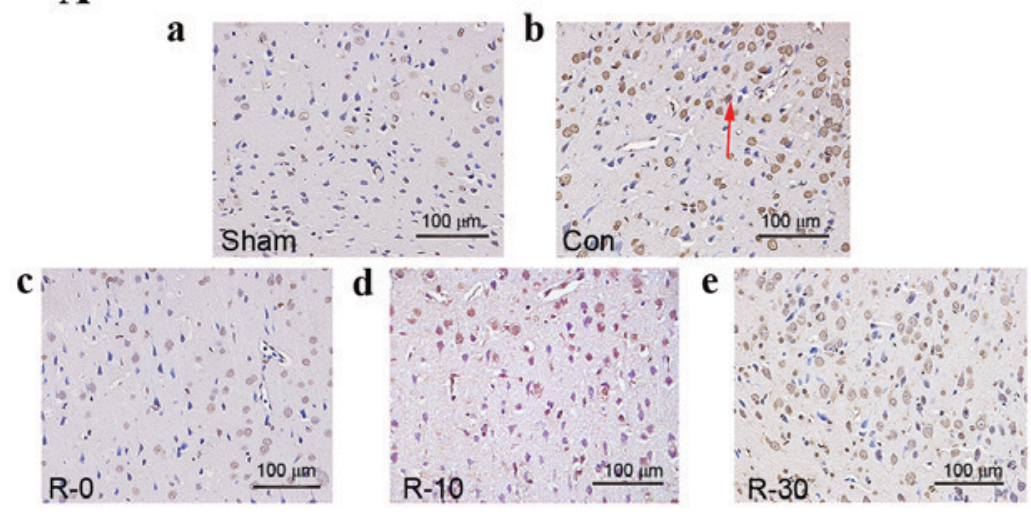

B

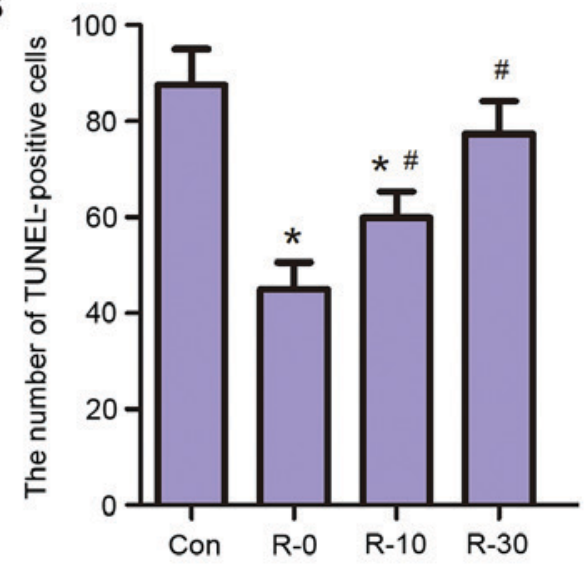

Figure 3. RIPostC reduced the number of TUNEL-positive cells following reperfusion (n=5/group). (A) Representative photomicrographs of TUNEL staining in the peri-infarct zone of rat brain sections from (a) sham, (b) control, (c) R-0, (d) R-10 and (e) R-30 groups at 24 h following reperfusion. The arrow indicates TUNEL-positive cells. Magnification, x400; scale bar, $100 \mu \mathrm{m}$. (B) Quantification of TUNEL-positive cells. Data are presented as the mean \pm standard deviation. ${ }^{*} \mathrm{P}<0.05$ vs. the control group; ${ }^{*} \mathrm{P}<0.05$ vs. R-0 group. Con, control; RIPostC, remote ischemic postconditioning; R-0, -10 , -30 , RIPostC 0 , 10 and 30 min groups, respectively; TUNEL, terminal deoxynucleotidyl transferase dUTP nick end labeling.

A

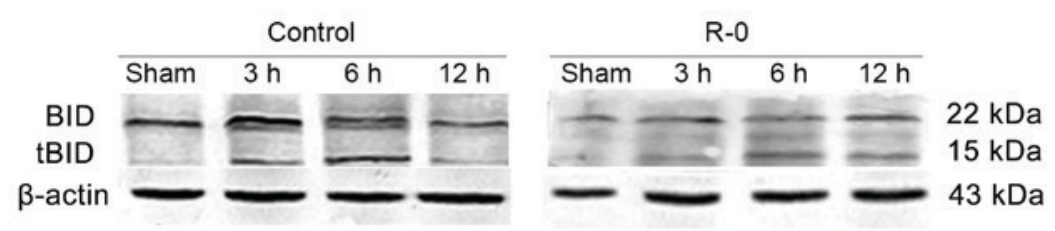

B

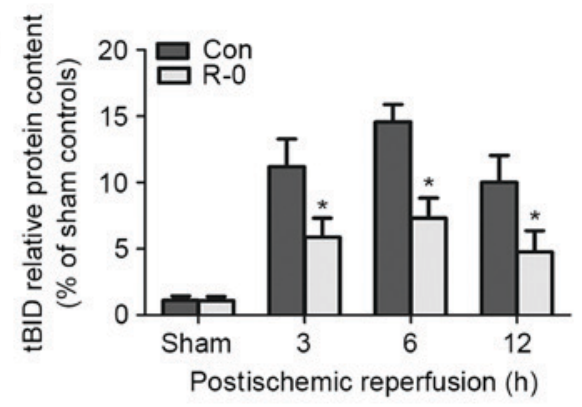

D

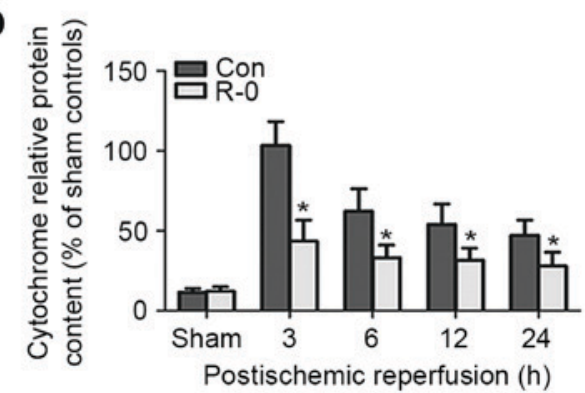

Figure 4. Remote ischemic postconditioning reduced BID activation and cytochrome $c$ release in ischemic brain samples (n=6). (A) Representative western blot analysis and (B) semi-quantification of western blot analysis of tBID at various time points. (C) Western blot analysis and (D) semi-quantification of western blot analysis of cytochrome $c$ at various time points. Equivalent loading in each lane was ensured by the detection of $\beta$-actin in the same tissue preparation. Data are presented as the mean \pm standard deviation. ${ }^{*} \mathrm{P}<0.05$ vs. the control group. BID, B-cell lymphoma 2 homology 3 interacting-domain death agonist; tBID, truncated BID; R-0, remote ischemic postconditioning 0 min group; Cyto $c$, cytochrome $c$; Con, control.

tBID level and translocation. The immunofluorescence signals of BID were very low in the non-ischemic cortex and indicated a diffusive pattern, consistent with its cytosolic distribution in normal cells. However, the signal increased following reperfusion, particularly $6 \mathrm{~h}$ after reperfusion, suggesting mitochondrial localization. Conversely, expression was weaker in the R-0 group compared with in the control group. Subcellular localization in the mitochodria was further confirmed by double-label staining with the anti-COXIV antibody. Furthermore, tBID was activated in the neurons as demonstrated by double-label staining with anti-NeuN antibody (a neuronal marker) (Fig. 6).

\section{Discussion}

Reperfusion following ischemia is often associated with induction of apoptosis-like cell death (15). The release of intramitochondrial contents into the cytosol is considered irreversible during the induction of apoptotic signaling (22). The present study demonstrated that RIPostC, applied as early as possible following reperfusion, may result in a significant neuroprotective effect in the rat MCAO model. The protective mechanisms of RIPostC may be associated with inhibition of the BID-mediated mitochondrial apoptotic pathway. 
A

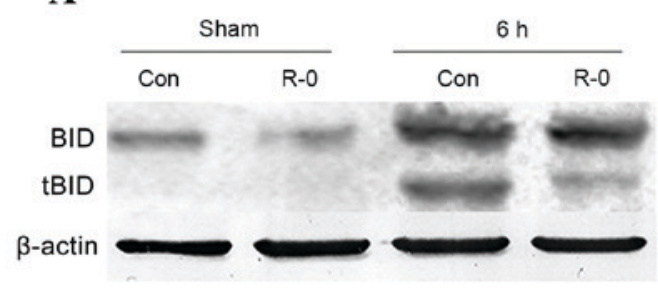

C

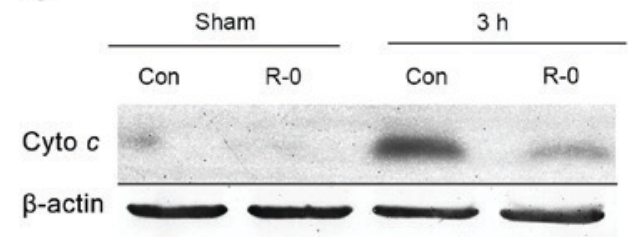

B

$22 \mathrm{kDa}$

$15 \mathrm{kDa}$

$43 \mathrm{kDa}$

D

$14 \mathrm{kDa}$

$43 \mathrm{kDa}$
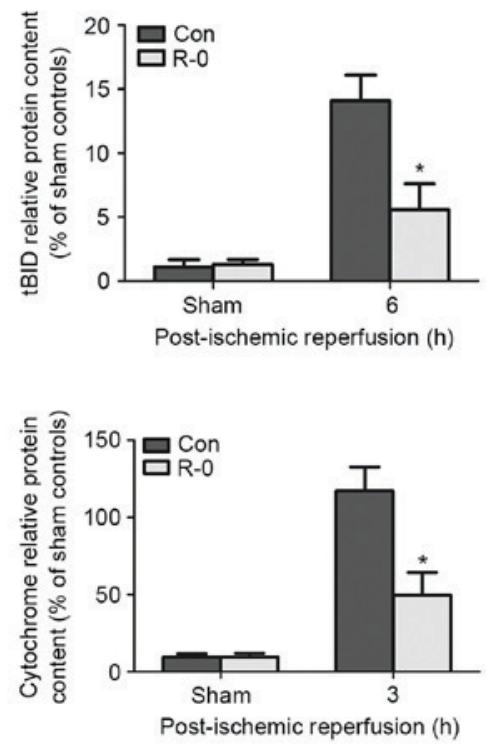

Figure 5. Expression peak of activated BID and cytochrome $c$ was decreased following RIPostC $(\mathrm{n}=6)$. (A) Western blot analysis and (B) semi-quantitative analysis of the effects of RIPostC on tBID peak expression. (C) Western blot analysis and (D) semi-quantitative analysis of the effects of RIpostC on cytochrome $c$ peak expression. Data are presented as the mean \pm standard deviation. $\mathrm{P}<0.05$ vs. the control group. RIPostC, remote ischemic postconditioning; BID, B-cell lymphoma 2 homology 3 interacting-domain death agonist; tBID, truncated BID; R-0, RIPostC 0 min group; Cyto $c$, cytochrome $c$; con, control.

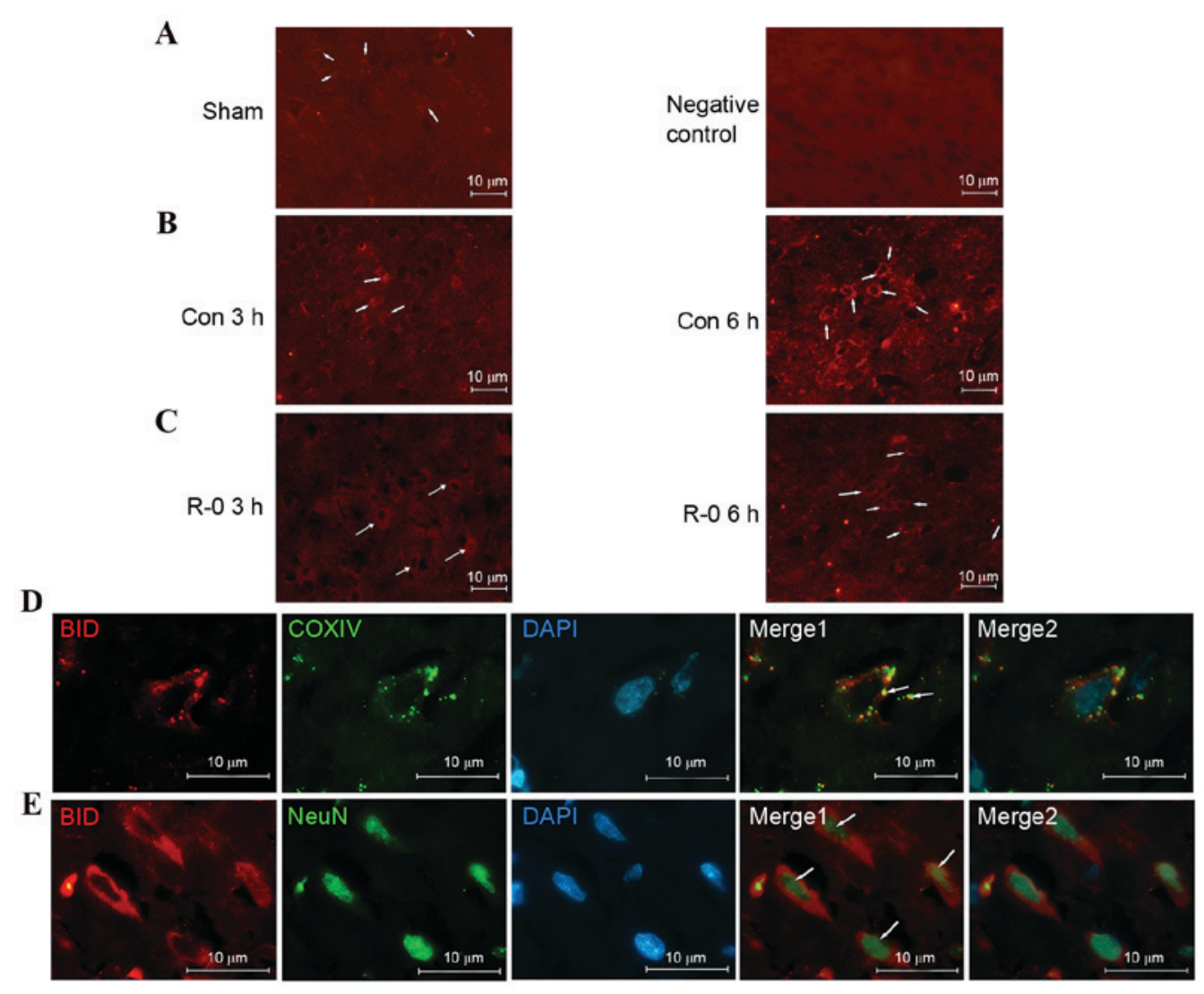

Figure 6. Activated BID is translocated to ischemic neurons following reperfusion. Representative immunofluorescent images of tBID obtained from the wild-type parietal cortex relevant to the infarct border zone. (A) Immunofluorescence signal of tBID was weak and diffusive in the non-ischemic cortex (left). A wild-type cortex staining control not incubated with the primary anti-BID antibody $6 \mathrm{~h}$ following reperfusion is presented on the right. (B) tBID immunofluorescence signal was increased at $3 \mathrm{~h}$ (left) and $6 \mathrm{~h}$ (right) following reperfusion and assumed a punctuated pattern. (C) tBID immunofluorescence signal appeared weaker at $3 \mathrm{~h}$ (left) and $6 \mathrm{~h}$ (right) following remote ischemic postconditioning. (A-C) Magnification, x400; scale bar, $10 \mu \mathrm{m}$. Arrows indicate tBID signals. (D) Double staining of tBID and mitochondria COXIV in cortical neurons from control group, $6 \mathrm{~h}$ following reperfusion. The overlapping image indicated that tBID was localized in the mitochondria (arrows mark the double-stained neurons). (E) Double staining of tBID and NeuN in the same treated cortex as D. The overlapping image indicated that enhanced tBID signals were detected in neurons (as marked by the arrows). (D and E) Magnification, x1,000; scale bar, $10 \mu \mathrm{m}$. BID, B-cell lymphoma 2 homology 3 interacting-domain death agonist; tBID, truncated BID; R- 0 , remote ischemic postconditioning 0 min group; COXIV, cytochrome $c$ oxidase IV; NeuN, neuronal nuclear antigen. 
Hess et al (23) reported that preclinical reports of remote limb conditioning in focal cerebral ischemia are promising; however, the underlying mechanism remains to be elucidated. At present, it is known that numerous mechanisms are involved: i) Humoral factors acting via systemic circulation; ii) neurogenic transmission with involvement of the autonomic nervous system; and iii) effects on circulating immune cells (24). However, a previous study involving the heart suggested that the pathway of protection in the target organ involves activation of kinases to resolve the reperfusion injury, or activation of survival factor enhancement pathways, which ultimately converge on the mitochondria to prevent opening of the mitochondrial permeability transition pore (25). The present study demonstrated that infarct volume, and the number of apoptotic cells, was significantly attenuated following administration of RIPostC; therefore, further investigations were conducted regarding the effects of RIPostC on the BID-mediated mitochondrial apoptotic pathway. The results appeared to be consistent with a previous study by Darling et al, which indicated that three cycles of occlusion-reperfusion in the first minute are optimal, and delaying postconditioning for even 1 min eliminates protection in the heart (26). The differing degree of protection between the results of the present study and other reports may reside in the differences in the duration of the ischemic insults and animal models. The results of the present study indicated that humoral factors may have a temporal profile of decay that reduces effectiveness, proportionate to the interval between ischemic stimulus and reperfusion, confirming that timing of the initiation of postconditioning is critical in the brain and heart.

As a $\mathrm{BH} 3$ domain-containing proapoptotic Bcl-2 family member, BID is inactive in the cytosol until exposed to various stress stimuli. tBID has been demonstrated to be important for the efficient recruitment of cytosolic Bax to the mitochondrial outer membrane (27), resulting in alterations to mitochondrial membrane permeability (7). In the present study, a western blot analysis detected tBID and cytochrome $c$ expression at the early stages of reperfusion. Translocation from the cytosol to mitochondria is considered an important activating mechanism for tBID and the propagation of extracellular to intracellular apoptotic signals (8), and this is consistent with the immunofluorescence results obtained. BID was activated and translocated to ischemic neuronal mitochondria following reperfusion. Numerous proapoptotic members of the Bcl-2 family may induce cytochrome $c$ release; however, tBID may initiate release of almost all mitochondrial cytochrome $c$ into the cytosol at a very low concentration compared with others (28). This may provide an explanation as to why its expression peak appears later than that of cytochrome $c$. These data suggested that BID is critical to the early activation of the mitochondrial apoptotic pathway.

Notably, BID activation and cytochrome $c$ release were not observed at the end of MCAO (data not shown). This is consistent with the previous findings, which suggested that induction of apoptosis is a delayed event (29). This delay may be attributable to the restoration of cellular energy levels during the reperfusion phase, which may be essential for the energy-requiring steps in the execution of the apoptotic program (30); similarly, proapoptotic substances produced during reperfusion may have contributed to the delay. Notably, in response to RIPostC, neither the inhibition of cytochrome $c$ release nor the neuroprotection against MCAO was complete, suggesting that the mitochondrial apoptotic pathway may have been activated by numerous mechanisms, or other factors were present resulting in reperfusion injury. However, BID bridges the activation of extrinsic and intrinsic apoptotic pathways in the early stage, due to its strategic position in the apoptotic signaling pathway, and may therefore be considered an ideal target for therapeutic intervention.

The present study used the bilateral femoral artery, as it is more practical to induce limb ischemia by placing a tourniquet on an extremity for a brief period. Therefore, RIPostC may have translational potential as a routine procedure in emergencies and in prehospital settings, and as a thrombolytic treatment in acute brain ischemia. However, the long-term effects of RIPostC were not observed, and it remains to be elucidated if underlying comorbidities may affect the efficacy of RIPostC; therefore, further studies are required.

In conclusion, the protective effects of RIPostC may be due to inhibition of the BID-mediated mitochondrial apoptotic pathway. The results of the present study suggested that RIPostC applied at the moment of reperfusion may exert a potent neuroprotective effect against focal cerebral ischemia/reperfusion injury. These data may help to elucidate the complex signaling cascades involved in the neuroprotective effects of RIPostC, leading to its potential future use as an effective treatment for stroke patients.

\section{Acknowledgements}

The present study was supported by the National Natural Science Foundation of China (grant no. 81271456).

\section{References}

1. Schaller B and Graf R: Cerebral ischemia and reperfusion: The pathophysiologic concept as a basis for clinical therapy. J Cereb Blood Flow Metab 24: 351-371, 2004.

2. Li J, Yu W, Li XT, Qi SH and Li B: The effects of propofol on mitochondrial dysfunction following focal cerebral ischemia-reperfusion in rats. Neuropharmacology 77: 358-368, 2014.

3. Li J, Han B, Ma X and Qi S: The effects of propofol on hippocampal caspase-3 and Bcl-2 expression following forebrain ischemia-reperfusion in rats. Brain Res 1356: 11-23, 2010.

4. Prasad SS, Russell M and Nowakowska M: Neuroprotection induced in vitro by ischemic preconditioning and postconditioning: Modulation of apoptosis and PI3K-Akt pathways. J Mol Neurosci 43: 428-442, 2011.

5. Zhang W, Wang B, Zhou S and Qiu Y: The effect of ischemic post-conditioning on hippocampal cell apoptosis following global brain ischemia in rats. J Clin Neurosci 19: 570-573, 2012.

6. Meller R, Cameron JA, Torrey DJ, Clayton CE, Ordonez AN, Henshall DC, Minami M, Schindler CK, Saugstad JA and Simon RP: Rapid degradation of Bim by the ubiquitin-proteasome pathway mediates short-term ischemic tolerance in cultured neurons. J Biol Chem 281: 7429-7436, 2006.

7. Wang $\mathrm{Y}$ and Tjandra N: Structural insights of tBid, the caspase-8-activated Bid, and its BH3 domain. J Biol Chem 288: 35840-35851, 2013.

8. Shamas-Din A, Bindner S, Zhu W, Zaltsman Y, Campbell C, Gross A, Leber B, Andrews DW and Fradin C: tBid undergoes multiple conformational changes at the membrane required for Bax activation. J Biol Chem 288: 22111-22127, 2013.

9. Tobaben S, Grohm J, Seiler A, Conrad M, Plesnila N and Culmsee C: Bid-mediated mitochondrial damage is a key mechanism in glutamate-induced oxidative stress and AIF-dependent cell death in immortalized HT-22 hippocampal neurons. Cell Death Differ 18: 282-292, 2011. 
10. Ding ZM, Wu B, Zhang WQ, Lu XJ, Lin YC, Geng YJ and Miao YF: Neuroprotective effects of ischemic preconditioning and postconditioning on global brain ischemia in rats through the same effect on inhibition of apoptosis. Int J Mol Sci 13: 6089-6101, 2012

11. Yan C, Chen J, Chen D, Minami M, Pei W, Yin XM and Simon RP: Overexpression of the cell death suppressor Bcl-w in ischemic brain: Implications for a neuroprotective role via the mitochondrial pathway. J Cereb Blood Flow Metab 20: 620-630, 2000.

12. Longa EZ, Weinstein PR, Carlson S and Cummins R: Reversible middle cerebral artery occlusion without craniectomy in rats. Stroke 20: 84-91, 1989

13. Garcia JH, Wagner S, Liu KF and Hu XJ: Neurological deficit and extent of neuronal necrosis attributable to middle cerebral artery occlusion in rats. Statistical validation. Stroke 26: 627-635, 1995.

14. Gao L, Ji X, Song J, Liu P, Yan F, Gong W, Dang S and Luo Y: Puerarin protects against ischemic brain injury in a rat model of transient focal ischemia. Neurol Res 31: 402-406, 2009.

15. Li X, Luo P, Wang F, Yang Q, Li Y, Zhao M, Wang S, Wang Q and Xiong L: Inhibition of N-myc downstream-regulated gene-2 is involved in an astrocyte-specific neuroprotection induced by sevoflurane preconditioning. Anesthesiology 121: 549-562, 2014

16. Wang Q, Peng Y, Chen S, Gou X, Hu B, Du J, Lu Y and Xiong L: Pretreatment with electroacupuncture induces rapid tolerance to focal cerebral ischemia through regulation of endocannabinoid system. Stroke 40: 2157-2164, 2009.

17. Wang KC, Koprivica V, Kim JA, Sivasankaran R, Guo Y, Neve RL and He Z: Oligodendrocyte-myelin glycoprotein is a Nogo receptor ligand that inhibits neurite outgrowth. Nature 417: 941-944, 2002

18. Wang Q, Zhang X, Ding Q, Hu B, Xie Y, Li X, Yang Q and Xiong L: Limb remote postconditioning alleviates cerebral reperfusion injury through reactive oxygen species-mediated inhibition of delta protein kinase C in rats. Anesth Analg 113: 1180-1187, 2011.

19. Gidday JM: Cerebral preconditioning and ischaemic tolerance. Nat Rev Neurosci 7: 437-448, 2006.
20. Zhou Y, Lekic T, Fathali N, Ostrowski RP, Martin RD, Tang J and Zhang JH: Isoflurane posttreatment reduces neonatal hypoxic-ischemic brain injury in rats by the sphingosine-1-phosphate/phosphatidylinositol-3-kinase/Akt pathway. Stroke 41: 1521-1527, 2010.

21. Dirnagl U, Simon RP and Hallenbeck JM: Ischemic tolerance and endogenous neuroprotection. Trends Neurosci 26: 248-254, 2003.

22. Peixoto PM, Teijido O, Mirzalieva O, Dejean LM, Pavlov EV, Antonsson B and Kinnally KW: MAC inhibitors antagonize the pro-apoptotic effects of tBid and disassemble Bax/Bak oligomers. J Bioenerg Biomembr 49: 65-74, 2017.

23. Hess DC, Hoda MN and Bhatia K: Remote limb perconditioning [corrected] and postconditioning: Will it translate into a promising treatment for acute stroke? Stroke 44: 1191-1197, 2013.

24. Weber C: Far from the heart: Receptor cross-talk in remote conditioning. Nat Med 16: 760-762, 2010.

25. Szijártó A, Czigány Z, Turóczi Z and Harsányi L: Remote ischemic perconditioning-a simple, low-risk method to decrease ischemic reperfusion injury: Models, protocols and mechanistic background. A review. J Surg Res 178: 797-806, 2012

26. Darling CE, Jiang R, Maynard M, Whittaker P, Vinten-Johansen J and Przyklenk K: Postconditioning via stuttering reperfusion limits myocardial infarct size in rabbit hearts: Role of ERK1/2. Am J Physiol Heart Circ Physiol 289: H1618-H1626, 2005.

27. Ott M, Norberg E, Zhivotovsky B and Orrenius S: Mitochondrial targeting of $\mathrm{tBid} / \mathrm{Bax}$ : A role for the TOM complex? Cell Death Differ 16: 1075-1082, 2009

28. Luo X, Budihardjo I, Zou H, Slaughter C and Wang X: Bid, a $\mathrm{Bcl} 2$ interacting protein, mediates cytochrome $\mathrm{c}$ release from mitochondria in response to activation of cell surface death receptors. Cell 94: 481-490, 1998.

29. Lipton P: Ischemic cell death in brain neurons. Physiol Rev 79: 1431-1568, 1999.

30. Leist M, Single B, Castoldi AF, Kühnle S and Nicotera P: Intracellular adenosine triphosphate (ATP) concentration: A switch in the decision between apoptosis and necrosis. J Exp Med 185: 1481-1486, 1997. 